\title{
Puerarin attenuates myocardial hypoxia/reoxygenation injury by inhibiting autophagy via the Akt signaling pathway
}

\author{
HUIXIONG TANG $^{1 *}$, XUDONG SONG $^{1 *}$, YUANNA LING $^{1}$, XIANBAO WANG ${ }^{1}$, \\ PINGZHEN YANG ${ }^{1}$, TAO LUO ${ }^{2}$ and AIHUA CHEN ${ }^{1}$ \\ ${ }^{1}$ Department of Cardiology, Zhujiang Hospital, Southern Medical University, Guangzhou, Guangdong 510282, P.R. China; \\ ${ }^{2}$ Division of Cardiology, Department of Medicine, University of California Irvine Medical Center, Orange, CA 92868, USA
}

Received December 22, 2015; Accepted February 1, 2017

DOI: $10.3892 / \mathrm{mmr} .2017 .6424$

\begin{abstract}
Puerarin (Pur), which is the major bioactive ingredient extracted from the root of Pueraria lobata (Willd.) Ohwi, has been demonstrated to relieve myocardial ischemia/ reperfusion (I/R) injury. Macroautophagy, or autophagy, is an evolutionarily conserved cellular catabolic mechanism that is involved in myocardial I/R injury. The present study evaluated the involvement of autophagy in the protective mechanisms of Pur during myocardial hypoxia/reoxygenation (H/R). The results revealed that Pur and 3-methyladenine pretreatment exerted a cardioprotective effect against H/R-induced cell viability loss. Pur also decreased the ratio of light chain 3 (LC3) -II/LC3-I and the degradation of p62 during H/R, which was accompanied by an increased level of phosphorylated-protein kinase B (Akt). These findings suggested that autophagy during myocardial H/R was inhibited by Pur, and this was further confirmed by the results of transmission electron microscopy and adenovirus-monomeric red fluorescent protein-green fluorescent protein-light chain 3 transfection. Furthermore, Pur inhibited the increased levels of autophagy induced by rapamycin, and the autophagy-inhibiting effects of Pur during myocardial $\mathrm{H} / \mathrm{R}$ were abolished by the Akt signaling inhibitor API-2. Collectively, these data indicate that Pur pretreatment
\end{abstract}

Correspondence to: Dr Aihua Chen, Department of Cardiology, Zhujiang Hospital, Southern Medical University, 253 Gongye Avenue, Guangzhou, Guangdong 510282, P.R. China

E-mail: zj_chenaihua@126.com

*Contributed equally

Abbreviations: Pur, puerarin; 3-MA, 3-methyladenine; Ra, rapamycin; API-2, Akt signaling inhibitor; 5-BrdU, 5-bromo-2'deoxyuridine; I/R, ischemia/reperfusion; H/R, hypoxia/reoxygenation; PI3K/Akt, phosphatidylinositol-3-kinase/protein kinase B; AMPK, 5' adenosine monophosphate-activated protein kinase; TEM, transmission electron microscope

Key words: puerarin, autophagy, hypoxia/reoxygenation injury, Akt, cardiomyocytes may attenuate myocardial $\mathrm{H} / \mathrm{R}$ injury by inhibiting autophagy via the Akt signaling pathway.

\section{Introduction}

Puerarin (Pur), which is the major bioactive ingredient extracted from the root of Pueraria lobata (Willd.) Ohwi, has been widely used in the treatment of cardiovascular diseases, cerebrovascular disorders and diabetes in China(1). Pur has been demonstrated to exert the following protective effects against myocardial ischemia/reperfusion (I/R) injury: Amelioration of oxygen consumption, restriction of the infarct area and improvement of diastolic function $(2,3)$. Previous studies have further demonstrated that these protective effects may be associated with the inhibition of mitochondrial permeability transition pore opening, activation of the mitochondrial adenosine triphosphate (ATP)-sensitive potassium channel, opening of the calcium-activated potassium channel and activation of protein kinase $\mathrm{C}(4,5)$. Although numerous studies have focused on the pharmacological properties of Pur, the mechanisms underlying the protective effects of Pur against myocardial I/R injury remain to be fully elucidated.

The mechanisms underlying myocardial I/R injury have traditionally been considered to include increased levels of reactive oxygen species, calcium overload and aberrant ATP production (6). However, previous research has also revealed that autophagy is involved in the mechanisms of myocardial I/R injury (7). Autophagy, by which mammalian cells degrade and recycle macromolecules and organelles, is vital for intracellular homeostasis at the basic level. It is activated to relieve adverse effects under various stress conditions, including nutritional deprivation, oxidative stress and ischemic injury (8). Previous studies have supported the suggestion that enhanced autophagy exerts protective effects during myocardial ischemia via protein clearance and restoration of cellular ATP levels $(9,10)$. However, during the reperfusion phase, increased autophagy in cardiomyocytes becomes a maladaptive process, which causes further myocardial damage and cell death $(7,11)$. Therefore, the manipulation of autophagy may be considered a novel method to relieve myocardial I/R injury.

The phosphatidylinositol-3-kinase/protein kinase B (PI3K/Akt) signaling pathway is critical for cell survival under 
stress conditions. Akt has previously been reported to be activated by Pur in various models, including cerebral I/R $(12,13)$. Furthermore, by activating Akt, autophagy can be negatively controlled (14). Given the important role of autophagy in myocardial I/R, the present study aimed to investigate whether Pur protects against myocardial I/R injury by inhibiting autophagy via the PI3K/Akt signaling pathway. The results of the present study provide further understanding of the protective mechanisms of Pur and the involvement of autophagy in myocardial I/R injury.

\section{Materials and methods}

Reagents. Pur (Fig. 1) was purchased from Tokyo Chemical Industry Co., Ltd. (Tokyo, Japan). Rapamycin (Ra), 3-methyladenine (3-MA), Akt signaling inhibitor (API-2) and 5-bromo-2'-deoxyuridine (5-BrdU) were purchased from Sigma-Aldrich Merck KGaA (Darmstadt, Germany). 3-(4,5-dimethylthiazol-2-yl)-5-(3-carboxymethoxyphenyl)-2(4-sulfophenyl) -2H-tetrazolium inner salt (MTS) was purchased from Promega Corporation (Madison, WI, USA). Dulbecco's modified Eagle's medium (DMEM) and fetal bovine serum (FBS) were purchased from Thermo Fisher Scientific, Inc. (Beijing, China). All antibodies for immunoblotting were purchased from Cell Signaling Technology, Inc. (Danvers, MA, USA), with the exception of the GAPDH antibody (KangChen Bio-tech, Inc., Shanghai, China) and goat anti-rabbit secondary antibody (Boster Systems, Inc., Pleasanton, CA, USA). Adenovirus-monomeric red fluorescent protein-green fluorescent protein-light chain 3 (Ad-mRFP-GFP-LC3) was purchased from HanBio Biotechnology Co., Ltd. (Shanghai, China). Neonatal Sprague Dawley rats ( 220; 1-2 days; 5-6 g) were obtained from the Experimental Animal Center of Southern Medical University (Guangzhou, China; license no. scxk-Guangdong-2006-0015) where they had been housed with female rats under standard conditions with a light/dark cycle of $12 \mathrm{~h}$ and fed by female rats.

Cell isolation and culture. Neonatal rat cardiomyocytes (NRCs) were isolated from the ventricular heart of 1-2 day old Sprague Dawley rats as previously described (15), following which the animals were immediately sacrificed by decapitation. The isolation procedure was performed in accordance with the Guide for the Care and Use of Laboratory Animals, published by the US National Institutes of Health (publication no. 85-23, revised 1996) and was approved by the Southern Medical University Experimental Animal Ethics Committee (Guangzhou, China). NRCs were plated at a density of $5 \times 10^{5}$ cells $/ 60 \mathrm{~mm}$ diameter plate, and cultured in DMEM supplemented with $10 \%$ FBS and $1 \%$ penicillin/streptomycin (the complete medium) in a $5 \% \mathrm{CO}_{2}$ humidified atmosphere at $37^{\circ} \mathrm{C}$. 5 - $\mathrm{BrdU}(0.1 \mathrm{mM})$ was added to the culture medium to inhibit fibroblast proliferation during the first $48 \mathrm{~h}$. The cells were subsequently cultured with the complete medium for a further $48 \mathrm{~h}$. The culture medium was changed daily.

Experimental treatment. Following culture for 96 h, NRCs were randomly divided into the following groups: The control group, in which cells were incubated under normal conditions; the hypoxia/reoxygenation $(\mathrm{H} / \mathrm{R})$ group, in which cells were

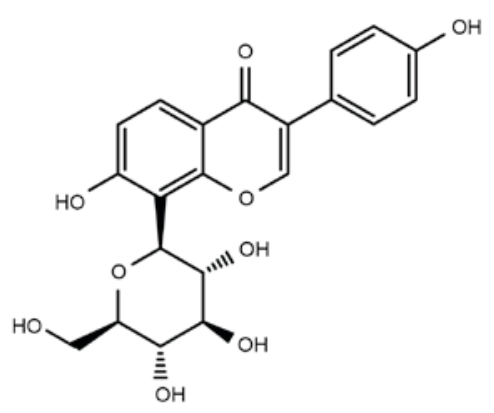

Figure 1. Chemical structure of puerarin.

exposed to $3 \mathrm{~h}$ hypoxia followed by $3 \mathrm{~h}$ reoxygenation; the $\mathrm{H} / \mathrm{R}+$ Pur group, in which cells were pretreated with Pur and were then exposed to H/R; H/R + 3-MA/Ra/API-2 group, in which cells were pretreated with 3-MA, Ra or API-2 and were then exposed to H/R; and the H/R + Pur + 3-MA/Ra/API-2 group, in which cells were pretreated with Pur plus 3-MA, $\mathrm{Ra}$ or API-2 and were then exposed to H/R. Pur $(50,100$ or $200 \mu \mathrm{M})(16), 3-\mathrm{MA}(5 \mathrm{mM}), \mathrm{Ra}(0.1 \mu \mathrm{M})$ and API-2 $(10 \mu \mathrm{M})$ were added to the culture medium $2 \mathrm{~h}$ prior to $\mathrm{H} / \mathrm{R}$.

Model of myocardial $H / R$. The myocardial H/R model was used to simulate I/R injury. For experiments under hypoxic conditions, NRCs wereincubated with phosphate-buffered saline (PBS) in a Modular Hypoxic Chamber (Billups-Rothenberg, Inc., Del Mar, CA, USA) saturated with $<0.1 \% \mathrm{O}_{2}, 95 \% \mathrm{~N}_{2}$ and $5 \% \mathrm{CO}_{2}$ at $37^{\circ} \mathrm{C}$ for $3 \mathrm{~h}$. Cells were subsequently subjected to reoxygenation by replacing PBS with the culture medium, and were returned to normoxic conditions for $3 \mathrm{~h}$ (17).

Cell viability. To assess cell viability, the MTS assay was used according to the manufacturer's protocol. NRCs were seeded in 96-well plates at a density of 5,000 cells/well. Following the indicated treatments, $20 \mu 1$ MTS solution was added to each well, and plates were subsequently incubated at $37^{\circ} \mathrm{C}$ for $3 \mathrm{~h}$. The optical density in each well was measured at $490 \mathrm{~nm}$ using a microplate reader (ELx800; BioTek Instruments, Inc., Winooski, VT, USA).

Western blot analysis. Western blotting was performed to determine LC3-II/LC3-I, p62, phosphorylated (p-)Akt (Ser473), Akt, P-5' adenosine monophosphate-activated protein kinase (p-AMPK) (Thr172), AMPK and GAPDH protein expression levels. Following the indicated treatments, cells were lysed using a total protein extraction kit (KangChen Bio-tech, Inc.) and a protease inhibitor cocktail (Thermo Fisher Scientific, Inc., Waltham, MA, USA). Lysates were subsequently centrifuged at $18,506 \times \mathrm{g}$ at $4^{\circ} \mathrm{C}$ for $15 \mathrm{~min}$. Protein concentration was determined using an enhanced bicinchoninic acid protein assay kit (Thermo Fisher Scientific, Inc.). The loading buffer was added and the protein samples were boiled in a water bath for $10 \mathrm{~min}$. Western blotting was performed according to standard procedures. Equal amounts of protein were resolved via SDS/PAGE (10 or 12\%) and transferred to PVDF membranes (Merck KGaA, Darmstadt, Germany). After blocking in 5\% non-fat milk at room temperature for $2 \mathrm{~h}$, membranes were incubated with the following primary antibodies overnight at $4^{\circ} \mathrm{C}$ : anti-LC3B (3868S), anti-p62 (8025S), anti-p-Akt (4060S), 

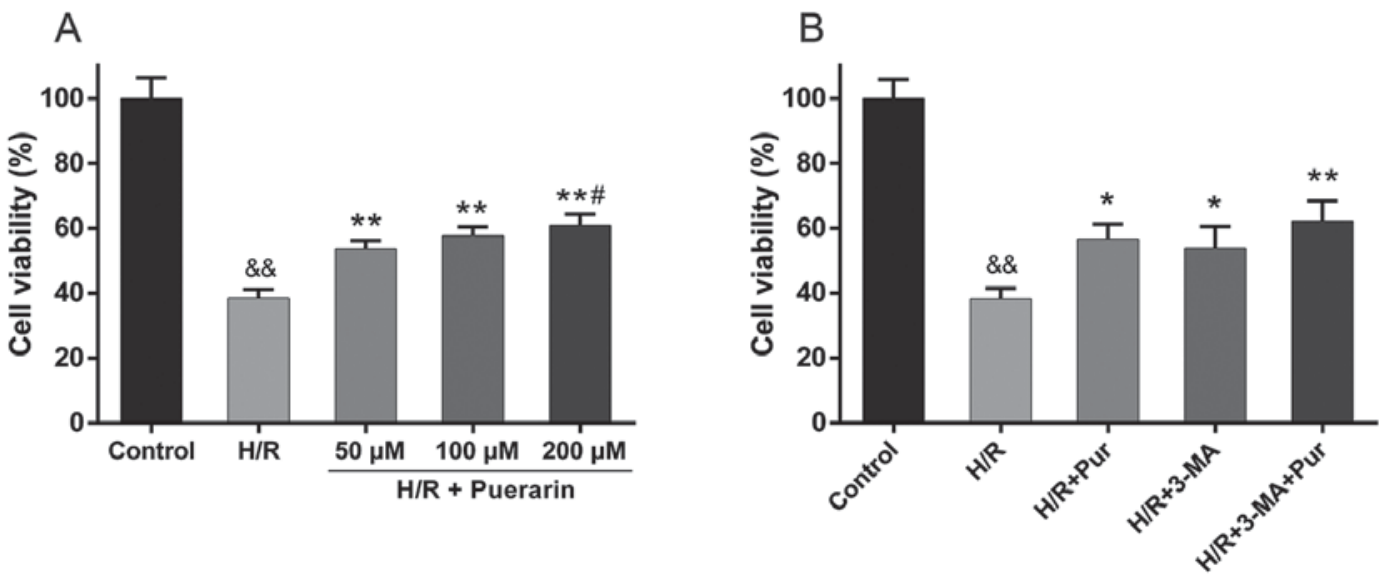

Figure 2. (A) Pur and (B) 3-MA protected neonatal rat cardiomyocytes from H/R-induced cell viability loss, as determined by MTS assay. Data are presented as the mean \pm standard deviation from five independent experiments. \& ${ }^{\&} \mathrm{P}<0.01$ vs. Control group; ${ }^{*} \mathrm{P}<0.05$ and ${ }^{* *} \mathrm{P}<0.01$ vs. H/R group; ${ }^{*} \mathrm{P}<0.05$ vs. $\mathrm{H} / \mathrm{R}+\mathrm{P} u r$ group (50 $\mu \mathrm{M})$. Pur, puerarin; 3-MA, 3-methyladenine; H/R, hypoxia/reoxygenation.

anti-Akt (4691P), anti-p-AMPK (2531S), anti-AMPK (2532S; all from Cell Signaling Technology, Inc.) and anti-GAPDH (KC-5G4; KangChen Bio-tech, Inc.). All dilutions were 1:1,000. After incubation for $2 \mathrm{~h}$ at room temperature with goat anti-rabbit secondary antibodies (1:8,000; BA1054; Boster Systems, Inc.), the bands were visualized using enhanced chemiluminescence (Merck KGaA). The blots were quantified by densitometry using Image-Pro Plus software version 6.0 (Media Cybernetics, Inc., Rockville, MD, USA) and the relative protein expression was compared with GAPDH.

Transmission electron microscopy (TEM). Following the indicated treatments, cells were washed, scraped, collected in a $1.5 \mathrm{ml}$ tube and centrifuged at $106 \mathrm{x} \mathrm{g}$ at $4^{\circ} \mathrm{C}$ for $5 \mathrm{~min}$. The cell pellets were subsequently fixed with $2.5 \%$ glutaraldehyde at $4^{\circ} \mathrm{C}$ overnight. Following fixation, cell pellets were dehydrated with a graded series of ethanol (50,70,90 and 100\%), propylene oxide and then infiltrated with a 1:1 mixture of propylene oxide and EMbed 812 . The samples were sliced into ultrathin sections $(75-80 \mathrm{~nm})$, which were stained and examined using a transmission electron microscope (JEM1200-EX; JEOL Ltd., Tokyo, Japan). In the samples, vesicles containing membranous structures (autophagosomes) in each cell were counted.

Ad-mRFP-GFP-LC3 transfection. Adenoviral transfection was performed according to the manufacturer's protocol. NRCs were seeded in $15 \mathrm{~mm}$ diameter glass-bottomed dishes at a density of 150 cells $/ \mathrm{mm}^{2}$ and $36 \mathrm{~h}$ later were incubated in DMEM containing 2\% FBS and adenovirus (multiplicity of infection of 15 ) at $37^{\circ} \mathrm{C}$ for $10 \mathrm{~h}$. The transfection medium was then replaced with the complete medium, and cells were further cultured at $37^{\circ} \mathrm{C}$ for $50 \mathrm{~h}$. Following the H/R treatments, cells were fixed with $4 \%$ paraformaldehyde and nuclei were stained with $2 \mu \mathrm{g} / \mathrm{ml}$ Hoechst 33342 (Sigma-Aldrich; Merck $\mathrm{KGaA}$ ) for $5 \mathrm{~min}$. Images of the cells were captured using a confocal fluorescence microscope (Olympus FV-1,000; Olympus Corporation, Tokyo, Japan). Autophagic flux was determined by evaluating the number of mRFP and GFP puncta/cell using Image-Pro Plus software version 6.0 (Media Cybernetics).
Statistical analysis. All experiments were performed $\geq 3$ times. SPSS 20.0 software (IBM SPSS, Armonk, NY, USA) was used for statistical analysis. The data were expressed as the mean \pm standard deviation. Statistical significance was analyzed by one-way analysis of variance followed by Tukey's post hoc test. $\mathrm{P}<0.05$ was considered to indicate a statistically significant difference.

\section{Results}

Pur and 3-MA protect cardiomyocytes from H/R-induced reductions in cell viability. To confirm the protective effect of Pur on myocardial I/R injury, an MTS assay was performed. Pur (50, 100 and $200 \mu \mathrm{M})$ was applied to NRCs $2 \mathrm{~h}$ prior to $\mathrm{H} / \mathrm{R}$. NRC viability was markedly decreased to $38.4 \pm 2.8 \%$ in the H/R group compared with the control group (Fig. 2A). However, NRC viability was significantly increased by 15.2-22.5\% following 50, 100 and $200 \mu \mathrm{M}$ Pur pretreatment compared with the H/R group (Fig. 2A), with the largest increase observed in the $200 \mu \mathrm{M}$ group, where NRC viability was significantly increased compared with the $50 \mu \mathrm{M}$ Pur pretreatment group (Fig. 2A). Accordingly, $200 \mu \mathrm{M}$ was selected as the concentration of Pur used in subsequent experiments.

3-MA is a classic autophagy inhibitor, which blocks autophagosome formation via the inhibition of type III PI3K, and was also tested in the myocardial H/R model. Treatment with 3-MA significantly increased NRC viability compared with the H/R group $(\mathrm{P}<0.05$; Fig. $2 \mathrm{~B})$, which was similar to the effect observed following Pur pretreatment. These results indicated that autophagy during myocardial H/R may contribute to cell death.

Pur inhibits H/R-induced autophagy in cardiomyocytes. To investigate the effects of Pur on H/R-induced autophagy in cardiomyocytes, western blot analysis was performed (Fig. 3A). As presented in Fig. 3B and C, H/R induced and increased the ratio of LC3-II/LC3-I, and decreased the expression levels of p62; however, Pur pretreatment $(50,100$ and $200 \mu \mathrm{M}$ ) decreased LC3-II/LC3-I ratio and increased 
A

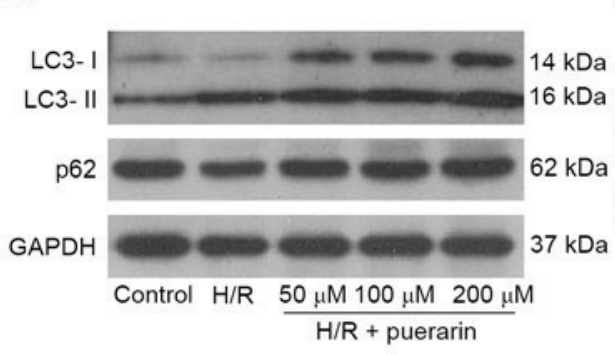

D

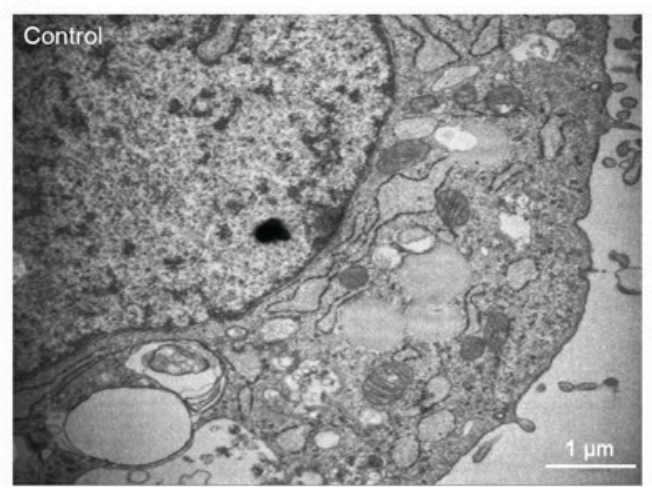

F

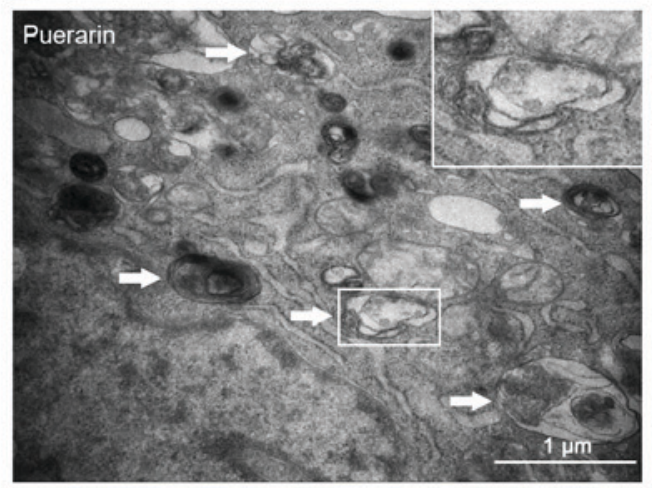

B

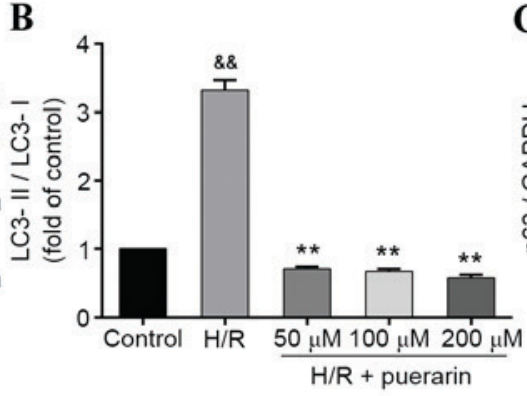

E

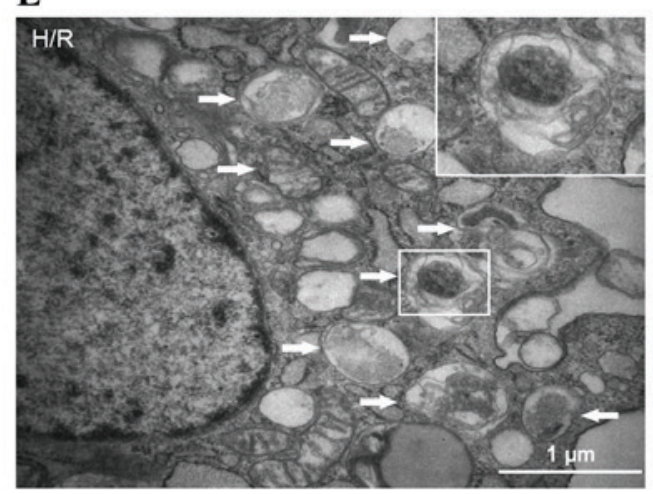

G

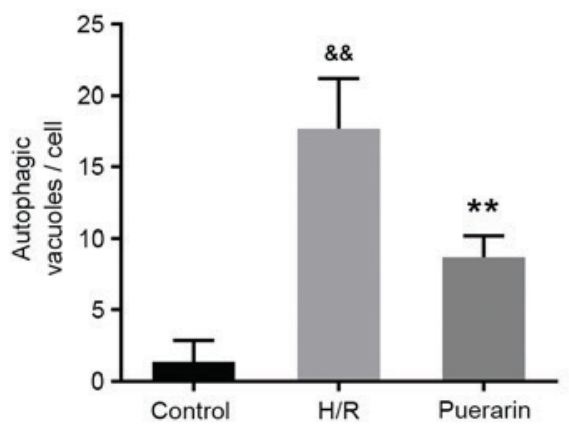

Figure 3. Pur inhibited H/R-induced autophagy in cardiomyocytes. (A) Autophagic marker proteins (LC3-II/LC3-I and p62) were examined by western blotting, and relative expression levels of (B) LC3-II/LC3-I and (C) p62 were semi-quantified. AVs were detected by transmission electron microscopy in (D) control, (E) H/R-treated and (F) Pur-pretreated/H/R-treated neonatal rat cardiomyocytes (scale bar: $1 \mu \mathrm{m}$ ). AVs are indicated by arrows. (G) Quantification of mean AVs per condition. Data are presented as the mean \pm standard deviation $(\mathrm{n}=3)$. ${ }^{\& \&} \mathrm{P}<0.01$ vs. Control group; ${ }^{* *} \mathrm{P}<0.01$ vs. $\mathrm{H} / \mathrm{R}$ group; ${ }^{*} \mathrm{P}<0.05$ vs. $\mathrm{H} / \mathrm{R}+$ Pur group $(50 \mu \mathrm{M})$. Pur, puerarin; H/R, hypoxia/reoxygenation; LC3, light chain 3; AVs, autophagic vacuoles.

p62 expression $(\mathrm{P}<0.01)$. These results suggested that Pur may inhibit H/R-induced autophagy in cardiomyocytes, which was confirmed by the results of TEM. As shown in Fig. 3E-F, large vesicles containing membranous structures (autophagosomes) appeared in the cytoplasm of cells in the $\mathrm{H} / \mathrm{R}$ group. Conversely, autophagosomes were scarce in the control group, which was characterized by normal cytoplasm, mitochondria, endoplasmic reticulum and nuclei. In addition, the number of autophagosomes induced by myocardial $H / R$ was significantly decreased by Pur pretreatment $(\mathrm{P}<0.01$; Fig. 3G).

To further investigate the inhibitory effects of Pur on autophagy, Ad-mRFP-GFP-LC3, a specific marker for autophagosomes and autolysosomes, was transfected into NSCs. The yellow puncta in merged pictures, as a result of merged m-RFP and GFP, represent autophagosomes, whereas the red puncta represent autolysosomes (Fig. 4A). Only a few yellow puncta appeared in the control group, whereas the number of yellow and red puncta visibly increased in the $H / R$ group (Fig. 4A). Following pretreatment with Pur, the number of yellow puncta (autophagosomes) significantly decreased by $60.1 \%$ compared with the $\mathrm{H} / \mathrm{R}$ group $(\mathrm{P}<0.01$; Fig. $4 \mathrm{~B})$. Conversely, there was no significant difference in the number of red puncta (autolysosomes) between the Pur group and H/R group (Fig. 4B).

Pur inhibits Ra-induced autophagy during myocardial H/R. $\mathrm{Ra}$ is one of the most commonly used drugs to stimulate autophagy. As aforementioned, Ra-mediated autophagy induction was performed in NRCs exposed to $\mathrm{H} / \mathrm{R}$, and western blotting was performed (Fig. 5A) The LC3-II/LC3-I ratio was significantly increased in the Ra group compared with the $\mathrm{H} / \mathrm{R}$ group $(\mathrm{P}<0.01$; Fig. $5 \mathrm{~B})$. However, following pretreatment with Ra + Pur, the LC3-II/LC3-I ratio was significantly 
A
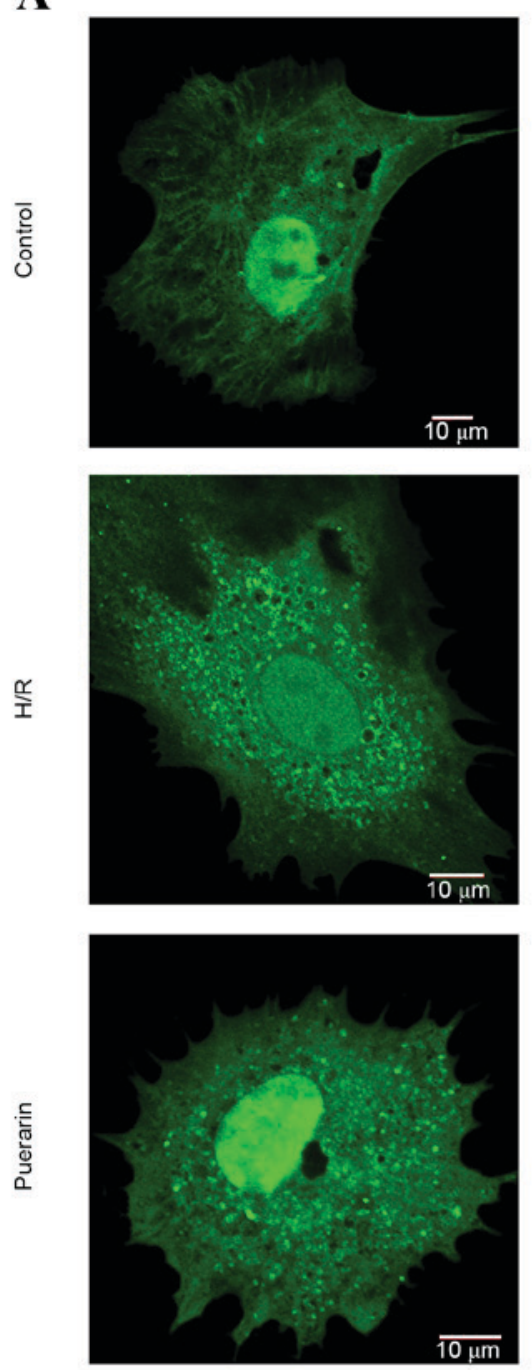

$\overline{10 \mu \mathrm{m}}$
mRFP
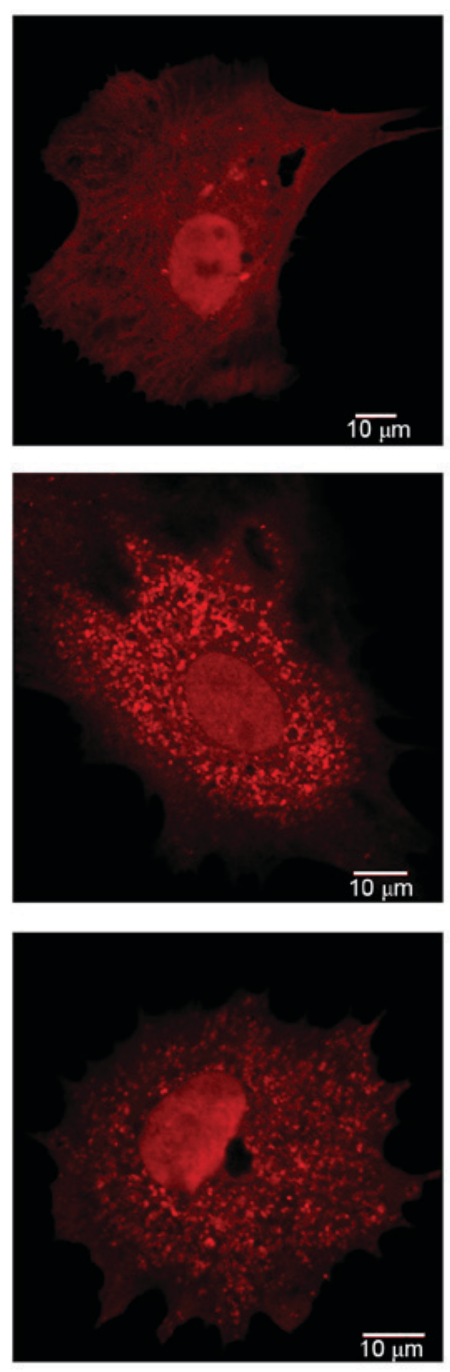

B
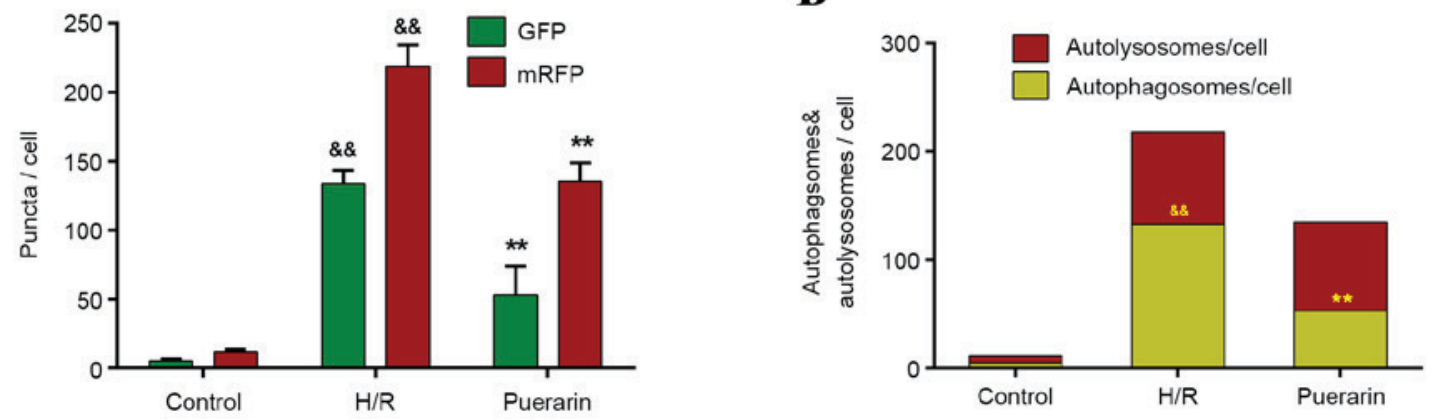

Figure 4. Puerarin inhibited autophagosome formation during myocardial H/R. (A) Confocal fluorescence microscopy analysis of neonatal rat cardiomyocytes transfected with adenovirus-mRFP-GFP-light chain 3 (scale bar: $10 \mu \mathrm{m}$ ), with quantification of GFP- and mRFP-labeled puncta. (B) Quantification of mean red and yellow fluorescent puncta. Data are presented as the mean \pm standard deviation $(n=3)$. \&\& $P<0.01$ vs. Control group; ${ }^{* *} P<0.01$ vs. H/R group. H/R, hypoxia/reoxygenation; mRFP, monomeric red fluorescent protein; GFP, green fluorescent protein.

decreased compared with the Ra group $(\mathrm{P}<0.01$; Fig. $5 \mathrm{~B})$ and the expression levels of p62 were elevated compared with the Ra group $(\mathrm{P}<0.01$; Fig. 5C). These results suggested that the increased autophagy induced by Ra during myocardial H/R was inhibited by Pur pretreatment.

Effects of Pur on the phosphorylation of Akt and AMPK during myocardial $H / R$. To investigate the potential molecular mechanisms involved in the autophagy-inhibiting effect of Pur during myocardial H/R, the PI3K/Akt and AMPK signaling pathways were assessed. The expression levels of p-Akt, Akt, p-AMPK and AMPK in groups were determined by western blotting. There was no significant difference in the ratios of p-Akt/Akt and p-AMPK/AMPK between the H/R group and the control group $(\mathrm{P}>0.05$; Fig. 5D and E). Pur pretreatment increased the phosphorylation of Akt compared with the H/R group $(\mathrm{P}<0.01$; Fig. 5D), but it did not significantly affect the phosphorylation of AMPK (Fig. 5E). 

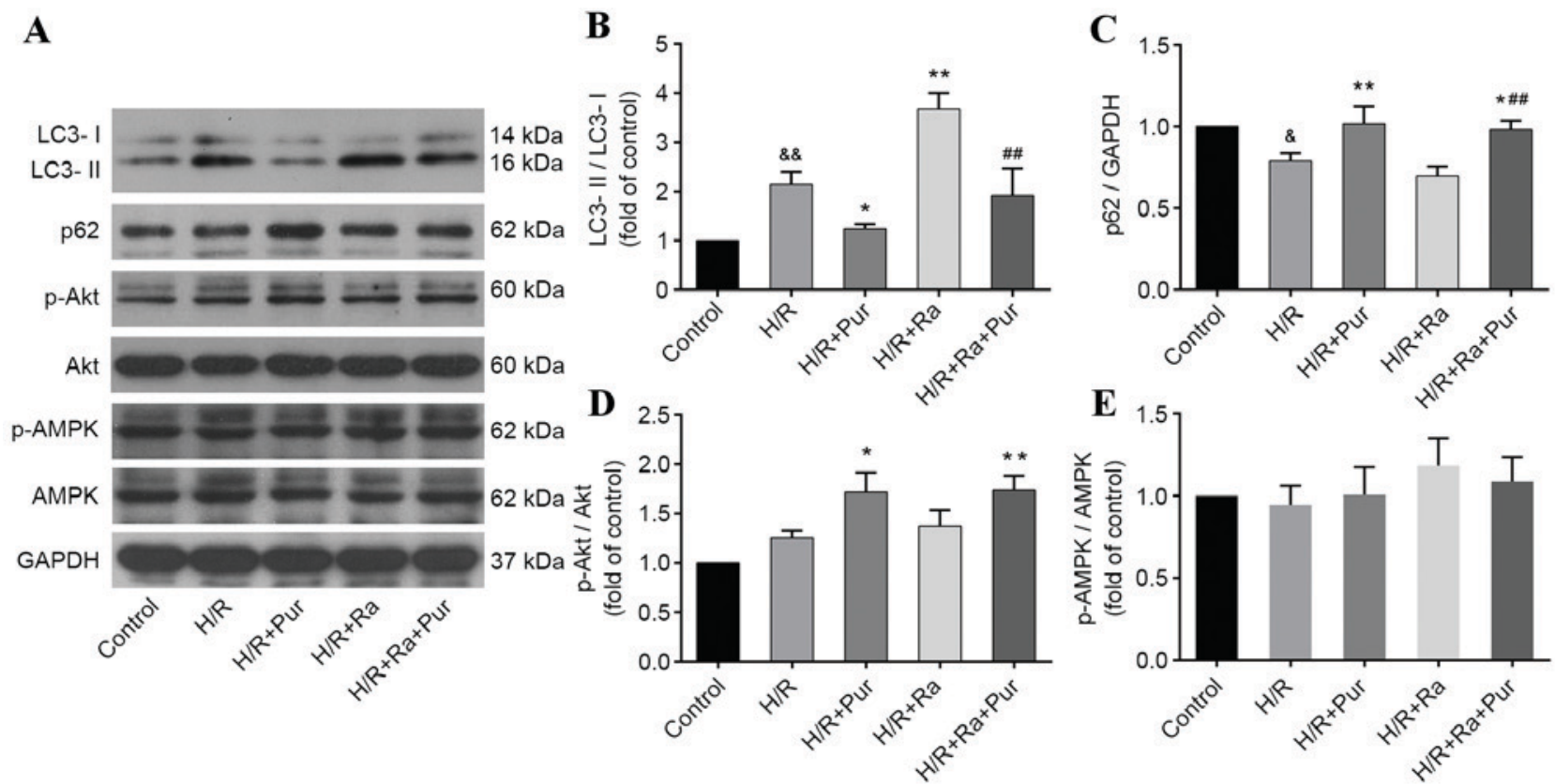

Figure 5. Pur inhibited Ra-mediated autophagy induction during H/R and increased Akt phosphorylation. (A) The results of western blotting and quantification of (B) LC3-II/LC3-I, (C) p62, (D) p-Akt/Akt and (E) p-AMPK/AMPK protein expression levels. Data are expressed as the mean \pm standard deviation. ${ }^{\text {P }}<0.05$ and ${ }^{\& \&} \mathrm{P}<0.01$ vs. Control group; ${ }^{*} \mathrm{P}<0.05$ and ${ }^{* *} \mathrm{P}<0.01$ vs. H/R group; ${ }^{\# \#} \mathrm{P}<0.01$ vs. $\mathrm{H} / \mathrm{R}+\mathrm{Ra}$ group. Pur, puerarin; Ra, rapamycin; H/R, hypoxia/reoxygenation; LC3, light chain 3; p-, phosphorylated; AMPK, 5' adenosine monophosphate-activated protein kinase.

A

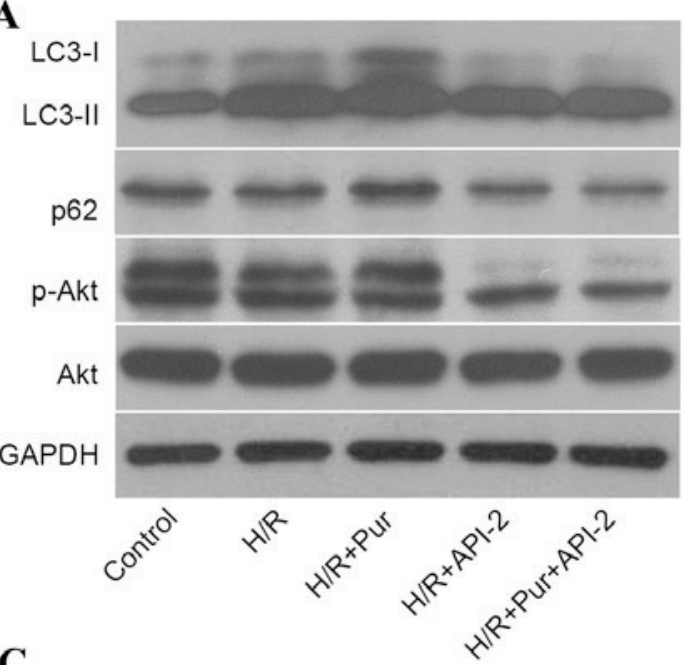

C

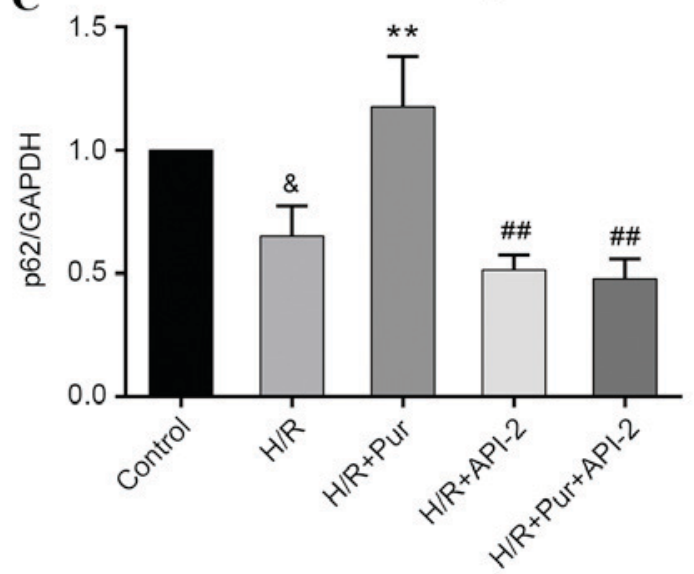

B

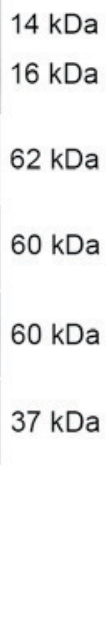

D
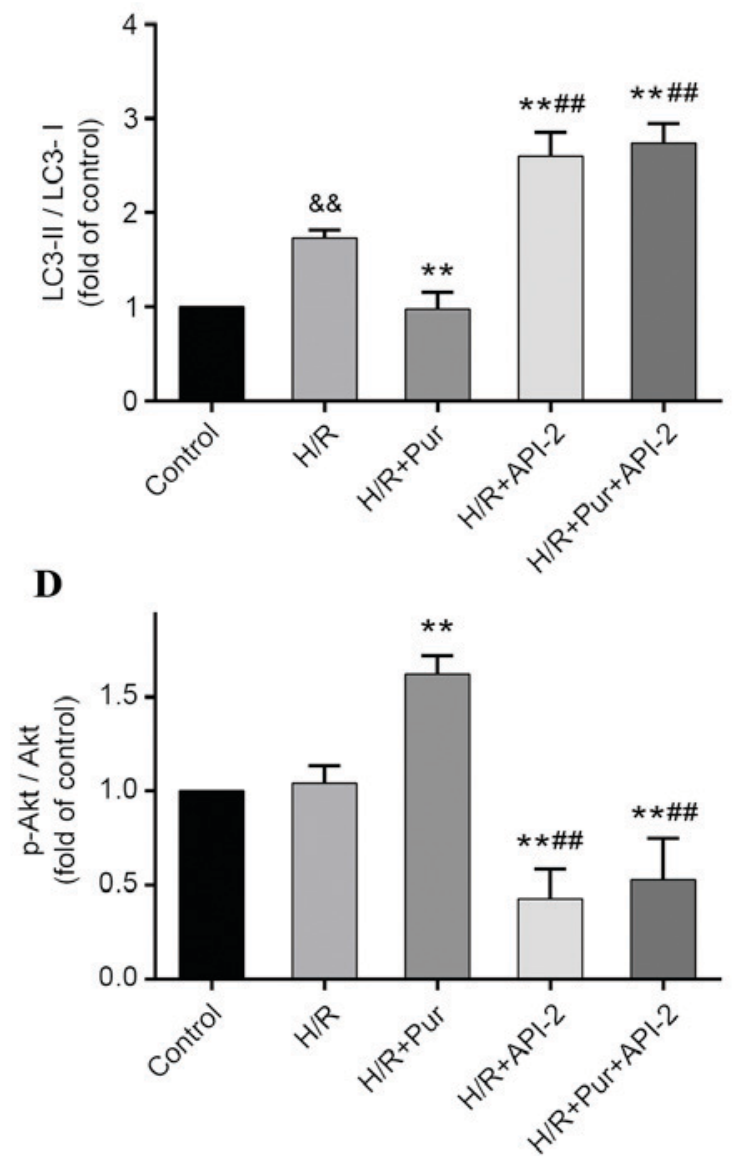

Figure 6. API-2 suppressed the autophagy-inhibiting effects of Pur by inhibiting Akt phosphorylation. (A) The results of western blotting and quantification of (B) LC3-II/LC3-I, (C) p62 and (D) p-Akt/Akt protein expression levels. Data are presented as the mean \pm standard deviation. ${ }^{\&} \mathrm{P}<0.05$ and \&\& $\mathrm{P}<0.01 \mathrm{vs}$.

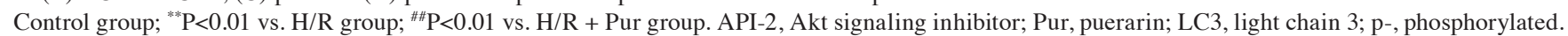


Autophagy-inhibiting effects of Pur during myocardial $H / R$ were suppressed by API-2. To further elucidate the involvement of the PI3K/Akt signaling pathway in the autophagy-inhibiting effects of Pur pretreatment during myocardial H/R, API-2 was applied to cells with or without Pur pretreatment and the effects were measured by western blotting (Fig. 6A). The LC3-II/LC3-I ratio was significantly increased in the H/R +API-2 + Pur group compared with the $\mathrm{H} / \mathrm{R}+$ Pur group $(\mathrm{P}<0.01$; Fig. $6 \mathrm{~B})$ and $\mathrm{p} 62$ protein expression levels were significantly decreased in the H/R + API-2 + Pur group compared with the H/R + Pur group $(\mathrm{P}<0.01$; Fig. 6C). Akt phosphorylation was significantly increased and autophagy was inhibited in the Pur group compared with the H/R group $(\mathrm{P}<0.01$; Fig. $6 \mathrm{~B}$ and $\mathrm{D})$; however, following pretreatment with Pur + API-2, Akt phosphorylation was significantly decreased and LC3-II/LC3-I ratio was increased compared with the $\mathrm{H} / \mathrm{R}+$ Pur group $(\mathrm{P}<0.01$; Fig. 6D). These findings indicated that the autophagy-inhibiting effects of Pur were abolished by API-2.

\section{Discussion}

Although Pur has been used in clinical practice in China for decades, the molecular mechanisms and targets underlying the pharmacological properties of Pur remain unclear, which limits its further clinical application (1). In the present study, the cardioprotective effects of Pur against H/R injury in NRCs were confirmed. Notably, Pur pretreatment inhibited autophagy during myocardial $\mathrm{H} / \mathrm{R}$, and this effect was abolished by inhibition of Akt signaling, indicating that Akt-dependent autophagy inhibition is involved in the protective mechanisms of Pur during myocardial H/R.

Autophagy is a dynamic and complex process wherein cytoplasm, protein aggregates and organelles are sequestered by double-membrane vesicles called autophagosomes, and trafficked to the lysosomes for degradation (18). LC3 and p62 are widely used autophagy markers. LC3 localizes to all types of autophagic membranes, and p62 protein is degraded by autophagy. Therefore, detecting the conversion of LC3-I to LC3-II and the degradation of $\mathrm{p} 62$ by western blotting has been widely used to monitor autophagic activity (19). In the present study, Pur was demonstrated to inhibit autophagy during myocardial $\mathrm{H} / \mathrm{R}$, which manifested as decreases in the ratio of LC3-II/LC3-I and the degradation of p62. As presented in Fig. 3A, the ratio of LC3-II/LC3-I was decreased in the Pur group due to a significant increase of LC3-I and a slight decrease of LC3-II protein expression levels, which suggests that the autophagy-inhibiting effects of Pur during $\mathrm{H} / \mathrm{R}$ are mainly due to the inhibition of autophagosome formation, and thus the conversion of LC3-I to LC3-II, rather than autophagosome degradation. To further confirm this, Ad-mRFP-GFP-LC3 transfection was performed. Consistent with previous observations, Pur pretreatment significantly inhibited autophagosome formation (Fig. 4C).

Autophagy can be either protective or detrimental, depending on the specific cellular context. It is activated as an adaptive response to stress conditions, including nutritional deprivation and ischemic injury (8). However, excessively activated autophagy during reperfusion may result in the loss of necessary proteins or organelles, leading to cellular dysfunction and autophagic cell death, which is detrimental for cell survival $(7,11,20)$. Therefore, inhibiting excessive autophagy may be a potential therapeutic strategy to attenuate myocardial I/R injury. Consistently, Huang et al (21) previously demonstrated that berberine exerts protective effects during myocardial I/R by suppressing autophagy (21). The results of the present study demonstrated that inhibition of autophagy by 3-MA reduced cell viability loss (Fig. 2B), which indicated that the increased autophagy response to $H / R$ is detrimental to cardiomyocytes. Therefore, by inhibiting autophagosome formation, Pur may maintain autophagic activity within a moderate range, thus exerting a protective effect against myocardial H/R injury.

Once the autophagy-inhibiting effects of Pur during myocardial $\mathrm{H} / \mathrm{R}$ were determined, the underlying mechanisms were explored further. It has previously been reported that Pur activates the PI3K/Akt signaling pathway in various models, including oxidative stress and cerebral I/R $(12,22)$. Consistent with these observations, the results of the present study demonstrated that Pur pretreatment increased Akt phosphorylation in cardiomyocytes exposed to H/R. Furthermore, the autophagy-inhibiting effects of Pur were abolished following treatment with an Akt signaling inhibitor, thus suggesting that Akt phosphorylation may be critical for the effects of Pur on autophagy inhibition. Combined with the inhibition of autophagosome formation by Pur, these findings indicated that Pur inhibits autophagy during myocardial H/R at the stage of induction via the Akt signaling pathway.

The PI3K/Akt signaling pathway is one of the most extensively studied pathways regulating autophagy (23). Previous studies have demonstrated that Akt activation inhibits autophagy by activating mammalian target of rapamycin complex 1 (mTORC1) (14), blocking the activation of forkhead box O3 (FOXO3) (24), or regulating the phosphorylation of Beclin1 (25). Ra inhibits mTORC1, which is one of the downstream targets of Akt, thereby activating autophagy (26). However, the autophagy-inhibiting effects of Pur were not suppressed by Ra in the present study (Fig. 5B), thus suggesting that the Akt-mTORC1-Atg1 is not the main pathway that mediates these effects. FOXO3 controls the transcription of autophagy-related genes, including LC3. Akt activation has been demonstrated to suppress FOXO3 activation, downregulate LC3 transcription and inhibit autophagy in skeletal muscle (24). However, the increased LC3-I levels observed in the Pur group do not support this hypothesis. Wang et al (25) demonstrated that Akt inhibits autophagy by mediating the phosphorylation of Beclin1 in tumor cells. Beclin1 is upregulated during the reperfusion phase (11), and it is essential for the PI3K complex, which is involved in the conversion of LC3-I to LC3-II (27). In combination with the findings of the present study and the aforementioned previous studies, there is a possibility that Pur inhibits autophagy by affecting the formation of the PtdIns3K complex, via Akt-mediated Beclin1 phosphorylation. Further investigation is required to test this hypothesis.

Pur has also been demonstrated to restore defective autophagy in the cardiac hypertrophy model and the ethanol-treated hepatocyte model via AMPK activation $(28,29)$. However, AMPK phosphorylation was not increased following pretreatment with Pur during myocardial 
H/R (Fig. 5E). During hypoxia and ischemia, AMPK acts as a sensor for energy deprivation and is activated by decreased ATP concentration. The energy crisis is resolved at the time of reperfusion, which in turn causes the inactivation of AMPK signaling (11). So the difference between models may be one explanation as to why AMPK phosphorylation was not increased following Pur pretreatment in the present study. The different intracellular environment may also enable Pur to act on the Akt signaling pathway and exert an inhibitory effect on autophagy.

In conclusion, the present study demonstrated that Pur pretreatment inhibited autophagy during myocardial $H / R$, and Akt-dependent autophagy inhibition may be one of the mechanisms underlying the protective effects of Pur against myocardial H/R injury. The results also suggested that excessive autophagy during the reperfusion phase is detrimental to cell survival. Taken together, the autophagy-inhibiting mechanism may be a basis for further clinical applications of Pur to relieve myocardial I/R injury.

\section{Acknowledgements}

The present study was supported by grants from the National Natural Science Foundation of China (grant no. 81270218, to Dr Chen), the National Natural Science Foundation of China (grant no. 81400190, to Dr Wang) and the Scientific and Technological Research Project of Guangzhou City (grant no. 2014A020212191, to Dr Yang).

\section{References}

1. Zhou YX, Zhang H and Peng C: Puerarin: A review of pharmacological effects. Phytother Res 28: 961-975, 2014.

2. Fan LL, Sun LH, Li J, Yue XH, Yu HX, Wang SY and Dong SQ: Protective effect of puerarin against myocardial reperfusion injury. Myocardial metabolism and ultrastructure. Chin Med J (Engl) 105: 451-456, 1992.

3. Pan HY, Gao Q, Yao H and Xia Q: The protective role and the mechanisms of puerarin on isolated rat heart during ischemia/ reperfusion. Zhongguo Ying Yong Sheng Li Xue Za Zhi 22: 455-459, 2006 (In Chinese).

4. Gao Q, Pan HY, Qiu S, Lu Y, Bruce IC, Luo JH and Xia Q: Atractyloside and 5-hydroxydecanoate block the protective effect of puerarin in isolated rat heart. Life Sci 79: 217-224, 2006

5. Gao Q, Yang B, Ye ZG, Wang J, Bruce IC and Xia Q: Opening the calcium-activated potassium channel participates in the cardioprotective effect of puerarin. Eur J Pharmacol 574: 179-184, 2007.

6. Murphy E and Steenbergen C: Mechanisms underlying acute protection from cardiac ischemia-reperfusion injury. Physiol Rev 88: 581-609, 2008.

7. Ma S, Wang Y, Chen Y and Cao F: The role of the autophagy in myocardial ischemia/reperfusion injury. Biochim Biophys Acta 1852: 271-276, 2015

8. Choi AM, Ryter SW and Levine B: Autophagy in human health and disease. N Engl J Med 368: 1845-1846, 2013.

9. Yan L, Vatner DE, Kim, SJ, et al: Autophagy in chronically ischemic myocardium. Proc Natl Acad Sci USA 102: 13807-13812, 2005.

10. Vilahur G, Juan-Babot O, Peña E, Oñate B, Casani L and Badimon L: Molecular and cellular mechanisms involved in cardiac remodeling after acute myocardial infarction. J Mol Cell Cardiol 50: 522-533, 2011.
11. Matsui Y, Takagi H, Qu X, Abdellatif M, Sakoda H, Asano T, Levine B and Sadoshima J: Distinct roles of autophagy in the heart during ischemia and reperfusion: Roles of AMP-activated protein kinase and Beclin 1 in mediating autophagy. Circ Res 100: 914-922, 2007.

12. Han JQ, Yu KY and He M: Effects of puerarin on the neurocyte apoptosis and p-Akt (Ser473) expressions in rats with cerebral ischemia/reperfusion injury. Zhongguo Zhong Xi Yi Jie He Za Zhi 32: 1069-1072, 2012 (In Chinese).

13. Wei SY, Chen Y and Xu XY: Progress on the pharmacological research of puerarin: A review. Chin J Nat Med 12: 407-414, 2014.

14. Arico S, Petiot A, Bauvy C, Dubbelhuis PF, Meijer AJ, Codogno P and Ogier-Denis E: The tumor suppressor PTEN positively regulates macroautophagy by inhibiting the phosphatidylinositol 3-kinase/protein kinase B pathway. J Biol Chem 276: 35243-35246, 2001.

15. Dong M, Ding W, Liao Y, Liu Y, Yan D, Zhang Y, Wang R, Zheng N, Liu S and Liu J: Polydatin prevents hypertrophy in phenylephrine induced neonatal mouse cardiomyocytes and pressure-overload mouse models. Eur J Pharmacol 746: 186-197, 2015.

16. Tang L, Liu D, Yi X, Xu T, Liu Y, Luo Y, Yin D and He M: The protective effects of puerarin in cardiomyocytes from anoxia/ reoxygenation injury are mediated by PKCE. Cell Biochem Funct 32: 378-386, 2014.

17. Ngoh GA, Facundo HT, Hamid T, Dillmann W, Zachara NE and Jones SP: Unique hexosaminidase reduces metabolic survival signal and sensitizes cardiac myocytes to hypoxia/reoxygenation injury. Circ Res 104: 41-49, 2009.

18. Mizushima $\mathrm{N}$ and Komatsu M: Autophagy: Renovation of cells and tissues. Cell 147: 728-741, 2011.

19. Jiang P and Mizushima N: LC3- and p62-based biochemical methods for the analysis of autophagy progression in mammalian cells. Methods 75: 13-18, 2015.

20. Valentim L, Laurence KM, Townsend PA, Carroll CJ, Soond S, Scarabelli TM, Knight RA, Latchman DS and Stephanou A: Urocortin inhibits Beclin1-mediated autophagic cell death in cardiac myocytes exposed to ischaemia/reperfusion injury. J Mol Cell Cardiol 40: 846-852, 2006.

21. Huang Z, Han Z, Ye B, Dai Z, Shan P, Lu Z, Dai K, Wang C and Huang W: Berberine alleviates cardiac ischemia/reperfusion injury by inhibiting excessive autophagy in cardiomyocytes. Eur J Pharmacol 762: 1-10, 2015.

22. Hwang YP and Jeong HG: Mechanism of phytoestrogen puerarin-mediated cytoprotection following oxidative injury: Estrogen receptor-dependent up-regulation of PI3K/Akt and HO-1. Toxicol Appl Pharmacol 233: 371-381, 2008.

23. Shanware NP, Bray K and Abraham RT: The PI3K, metabolic, and autophagy networks: Interactive partners in cellular health and disease. Annu Rev Pharmacol Toxicol 53: 89-106, 2013.

24. Mammucari C, Milan G, Romanello V, Masiero E, Rudolf R, Del Piccolo P, Burden SJ, Di Lisi R, Sandri C, Zhao J, et al: FoxO3 controls autophagy in skeletal muscle in vivo. Cell Metab 6: 458-471, 2007

25. Wang RC, Wei Y, An Z, Zou Z, Xiao G, Bhagat G, White M, Reichelt $J$ and Levine B: Akt-mediated regulation of autophagy and tumorigenesis through Beclin 1 phosphorylation. Science 338: 956-959, 2012.

26. Manning BD and Cantley LC: AKT/PKB signaling: Navigating downstream. Cell 129: 1261-1274, 2007.

27. Yue $\mathrm{Z}$ and Zhong Y: From a global view to focused examination: Understanding cellular function of lipid kinase VPS34-Beclin 1 complex in autophagy. J Mol Cell Biol 2: 305-307, 2010.

28. Liu B, Wu Z, Li Y, Ou C, Huang Z, Zhang J, Liu P, Luo C and Chen M: Puerarin prevents cardiac hypertrophy induced by pressure overload through activation of autophagy. Biochem Biophys Res Commun 464: 908-915, 2015.

29. Noh BK, Lee JK, Jun HJ, Lee JH, Jia Y, Hoang MH, Kim JW, Park KH and Lee SJ: Restoration of autophagy by puerarin in ethanol-treated hepatocytes via the activation of AMP-activated protein kinase. Biochem Biophys Res Commun 414: 361-366, 2011. 